\title{
Produção científica sobre nutrição no tratamento do câncer: estudo bibliométrico
}

\author{
Scientific production on nutrition in the treatment of cancer: bibliometric study
}

Producción científica sobre nutrición en el tratamiento del cáncer: estudio bibliométrico

Quéfren Guimarães Suzuki" ${ }^{1 *}$ Brenner Kássio Ferreira de Oliveira1, Maxwell Arouca da Silva ${ }^{1}$, Alessandra da Silva Carvalho ${ }^{1}$, Amanda da Silva Melo ${ }^{1}$, Cliviane Farias Cordeiro ${ }^{1}$, Priscilla Mendes Cordeiro'.

\section{RESUMO}

Objetivo: Investigar os índices de produções científicas de nutrição voltada para o tratamento do câncer no período de 2008 a 2018. Métodos: Trata-se de um estudo bibliométrico, quantitativo, do tipo descritivo da literatura científica. Na busca utilizou-se os descritores "nutrição" e "câncer" nas bases de dados LILACS, SCIELO e MEDLINE. Os dados obtidos foram analisados através da planilha Excel. Resultados: Dos 99 artigos analisados, os autores mais produtivos foram nutricionistas, com grau de doutorado, docentes, a maioria vinculados a instituições de ensino superior (IES) públicas, sendo a maior produtividade advinda de artigos da região Sudeste, com estratos qualis B1 e B2, destacando-se a Revista de Brasileira de Cancerologia. Os descritores estado nutricional e neoplasia foram os mais utilizados, bem como assuntos mais discorridos estavam os relacionados ao estado nutricional ou risco nutricional dos pacientes e a neoplasia maligna no trato gastrointestinal. Considerações finais: A nutrição garante suporte durante o tratamento do câncer. O conhecimento é embasado através de produções científicas. Quanto mais se produz conhecimento escrito, mais o conhecimento é disseminado à sociedade. Desta maneira, desenvolver pesquisas sobre a nutrição e câncer expõe a importância no contexto terapêutico do paciente, a fim de melhorar sua saúde.

Palavras-Chave: Bibliometria, Nutrição, Neoplasia maligna.

\section{ABSTRACT}

Objective: To investigate the scientific production indexes of nutrition focused on cancer treatment from 2008 to 2018. Methods: This is a bibliometric, quantitative study, of the descriptive type of scientific literature. In the search, the descriptors "nutrition" and "cancer" were used in the LILACS, SCIELO and MEDLINE databases. The data obtained were analyzed using the Excel spreadsheet. Results: Of the 99 articles analyzed, the most productive authors were nutritionists, with doctoral degrees, teachers, most of them linked to public higher education institutions (HEls), with the highest productivity coming from articles in the Southeast region, with qualifying strata B1 and B2, highlighting the Revista de Brasileira de Cancerologia. The descriptors nutritional status and neoplasia were the most used, as well as the most discussed subjects were those related to the nutritional status or nutritional risk of patients and malignant neoplasm in the gastrointestinal tract. Final considerations: Nutrition guarantees support during cancer treatment. Knowledge is based on scientific production. The more written knowledge is produced, the more knowledge is disseminated to society. In this way, developing research on nutrition and cancer exposes the importance in the patient's therapeutic context, in order to improve their health.

Key words: Bibliometry, Nutrition, Malignant neoplasm.

1 Universidade Federal do Amazonas (UFAM), Coari - AM. *E-mail: quefrensuzuki@hotmail.com 


\section{RESUMEN}

Objetivo: Investigar los índices de producción científica de nutrición centrados en el tratamiento del cáncer desde 2008 hasta 2018. Métodos: Este es un estudio bibliométrico, cuantitativo, del tipo descriptivo de literatura científica. En la búsqueda, los descriptores "nutrición" y "cáncer" se utilizaron en las bases de datos LILACS, SCIELO y MEDLINE. Los datos obtenidos se analizaron utilizando la hoja de cálculo Excel. Resultados: De los 99 artículos analizados, los autores más productivos fueron nutricionistas, con doctorados, docentes, la mayoría de ellos vinculados a instituciones públicas de educación superior (IES), con la mayor productividad proveniente de artículos en la región sudeste, con estratos B1 calificativos y B2, destacando la Revista de Brasileira de Cancerologia. Los descriptores del estado nutricional y la neoplasia fueron los más utilizados, así como los temas más discutidos fueron los relacionados con el estado nutricional o el riesgo nutricional de los pacientes y la neoplasia maligna en el tracto gastrointestinal. Consideraciones finales: La nutrición garantiza el apoyo durante el tratamiento del cáncer. El conocimiento se basa en la producción científica. Cuanto más conocimiento escrito se produce, más conocimiento se difunde a la sociedad. De esta manera, el desarrollo de investigaciones sobre nutrición y cáncer expone la importancia en el contexto terapéutico del paciente para mejorar su salud.

Palabras clave: Bibliometría, Nutrición, Neoplasia maligna.

\section{INTRODUÇÃO}

O estudo da neoplasia maligna ganhou destaque no campo da saúde pública e comunidade científica por ser uma das principais causas de mortalidade no mundo e a segunda causa de óbitos no Brasil (ABRAHÃO SA e MACHADO EC, 2014; BRASIL, 2017). Segundo a Estatistica de 2016 do Instituto Nacional do Câncer (2019), a mortalidade chegou em 212,27 mil casos, sendo 112,771 para homens e 99,499 para mulheres e, estimou-se para o biênio 2018-2019 a ocorrência de 600 mil novos casos da doença (BRASIL, 2017).

A etiologia para a neoplasia decorre de vários agentes causadores, isso inclui exposição a diversas causas externas (meio ambiente) e internas (hormônios, condições imunológicas e mutações genéticas) sendo a interação entre as duas causas a forma mais comum para o desenvolvimento. Entre os principais fatores carcinogênicos encontram-se a alimentação, fumo, álcool, obesidade, sedentarismo, contato frequente com carcinógenos, exposição a radiação, idade, etnia e sexo (BUONO HC, et al., 2017; BRASIL, 2017).

Logo, percebe-se que a alimentação aparece como um dos fatores externos que ocasiona esta doença, assim sendo, pode atuar nos estágios de iniciação, promoção e propagação do câncer, destacando-se entre outros fatores de risco (GARÓFOLO A, et al., 2004). A escolha do alimento está relacionada aos hábitos alimentares de cada um, então o consumo excessivo de alimentos industrializados e processados, por exemplo, pode desencadear lesões no organismo, aumentando as chances do desenvolvimento de neoplasias ao longo do tempo (AZEVEDO CD, DAL BOSCO SM, 2011).

No entanto, de outro ponto de vista, a alimentação saudável ajuda na prevenção ou mesmo atrasa o seu surgimento e, principalmente, durante o tratamento da doença em que a maioria dos quimioterápicos ocasiona efeitos indesejáveis a conduta dietoterápica minimiza os efeitos colaterais (DUTRA e SAGRILLO, 2014). Alimentos como frutas e hortaliças têm assumido posição de destaque nos estudos que envolvem a prevenção do câncer que, por meio de evidências epidemiológicas pode ser observado o efeito protetor contra essa patologia (GARÓFOLO A, et al., 2004). Esses alimentos devem fazer parte da alimentação diária, por serem considerados antioxidantes, devido a ação protetora efetiva contra os processos oxidativos que ocorrem no organismo e serem capazes de estabilizar ou desativar os radicais livres do organismo (AZEVEDO CD, DAL BOSCO SM, 2011).

É importante que a assistência nutricional atue em todos os estágios da doença, sendo imprescindível sua integração na estratégia terapêutica, fundamentada através de literaturas que garantem um bom acompanhamento nutricional e viabilizando a modulação sintomática através da diminuição das infecções e complicações pós-operatórias, menor tempo de hospitalização, melhor retorno às terapêuticas e modulação 
da resposta imunológica (CARVALHO G, et al., 2011). Nota-se a grande influência e o importante papel que a literatura científica assume como disseminadora de conhecimento. Barreto ML (2013), explica que toda ciência é difundida de forma escrita e todo novo conhecimento científico necessita ser escrito, revisto, comentado e verificado.

O estudo que analisa quantitativamente os documentos científicos em áreas específicas de produção científica pesquisada é a Bibliometria. Sua finalidade de gerar dados estatísticos estimando a colaboração da produção e disseminação do conhecimento científico de modo claro sobre qualquer área de estudos (SANTOS GC, 2015; SILVA FQ, et al., 2016; SOARES PB, et al., 2016). Esses dados podem ser utilizados na representação das tendências de pesquisa e identificação de temas para novas (SOARES PB, et al., 2016). Através da comunicação escrita é possível identificar, por exemplo, os periódicos mais produtivos, visualizar o que está sendo publicado e quais conhecimentos estão sendo construídos em determinada área (SILVA FQ, et al., 2016; SOARES PB, et al., 2016).

Desta maneira, a partir das diversas publicações sobre o assunto e julgando a carência de pesquisas sobre a análise da produção científica na temática, este trabalho buscou realizar um estudo bibliométrico com o objetivo de investigar os índices de produções científicas acerca da nutrição relacionada ao tratamento do câncer considerando a quantidade de artigos indexados em bases de dados, a instituição mais produtora e o quantitativo periódicos/ano com o tema no período de 2008 a 2018.

\section{MÉTODOS}

Trata-se de um estudo bibliométrico, quantitativo do tipo descritivo da literatura científica nacional desenvolvida por profissionais brasileiros sobre artigos que tratam da nutrição voltada para o câncer com ênfase nos últimos 10 anos. A bibliometria se apresenta com relevância para análise de produção científica de um país retratando o comportamento e o desenvolvimento de uma área de conhecimento específico (DEMO G, et al., 2017). A pesquisa foi realizada nas bases de dados Literatura Latino-Americana e do Caribe em Ciências da Saúde (LILACS), Scientific Eletronic Library Online (SCIELO) e MEDLINE, disponível na Biblioteca Virtual em Saúde (BVS).

A busca foi realizada nos meses de Agosto de 2018 a Março de 2019, para a coleta de dados foi realizado o pareamento dos descritores "nutrição" e "câncer" disponível no banco nacional de descritores (DeCS). Aplicaram-se os filtros ano de publicação de 2008 a 2018, com o tipo de documento, optando apenas por artigos e país de afiliação Brasil. Foram excluídas publicações que não contemplavam a temática proposta, publicações de características clínicas e relacionados à epidemiologia do câncer, nutrição associado a outras patologias, documentos de teses, monografias, comunicados e trabalhos apresentados em congressos, outros que não se enquadravam a busca nutrição e câncer, artigos sem acesso livre e artigos não encontrados (not found) na página direcionada disponível na base de dados, dessa forma, mesmo se tratando do tema nutrição e câncer através do título e resumo, os mesmos não puderam ser contabilizados pela carência de dados. Incluíram-se Artigos Originais, Artigos de Revisão e Relatos de Casos, pesquisas realizadas no Brasil e no idioma português e inglês de autores brasileiros.

Posterior a leitura dos títulos, resumos e palavras-chaves dos artigos incluídos na pesquisa, foram arquivados em pastas separados por base de dados e reavaliados em uma nova triagem, artigos repetidos permaneceram na base de dados com resultados em maior número e os artigos com pesquisas realizadas fora do Brasil foram excluídos. Em seguida, os artigos incluídos foram tabulados na planilha eletrônica da Microsoft Excel, por periódico, título do artigo, autores, ano de publicação, formação profissional, titulação acadêmica, representação da instituição (universidade, hospital, instituto e clínica) e estado/região de origem das publicações (baseado no primeiro autor), tipo de documento (artigo original, revisão e relato de caso), idioma e estratos Qualis (A1, A2, B1, B2, B3, B4, B5 e C).

Para encontrar os periódicos mais produtivos nesta busca, a partir dos dados encontrados foi aplicado a Lei de Bradford que, de acordo com Araújo CA (2006), objetiva descobrir a extensão na qual artigos de um assunto científico específico aparecem em periódicos destinados a temas gerais e não ao abordado 
exclusivamente, estudando a distribuição dos artigos em termos de variáveis de proximidade ou de afastamento. Aos artigos selecionados que não citavam a formação profissional ou a titulação do autor, optouse por fazer buscas no currículo Lattes dessas informações, à vista disso, considerou-se a compatibilidade das informações do currículo ao ano que descrevia o artigo.

\section{RESULTADOS E DISCUSSÃO}

$\mathrm{Na}$ etapa inicial do processo de busca foram identificadas 385 publicações científicas totais a partir da busca nas três bases de dados, sendo 212 artigos na base de dados LILACS, 45 artigos na SCIELO e 128 artigos na MEDLINE. Usando os critérios de inclusão e exclusão chegou ao resultado de cada base de dados: 67 artigos no LILACS, 7 artigos SCIELO e 25 artigos MEDLINE, totalizando 99 artigos selecionados para o estudo.

A formação profissional dos autores mostra que $78,8 \%$ destes foram produzidos por nutricionistas, no entanto outros profissionais como enfermeiro $(3,0 \%)$, médico $(2,0 \%)$ e fisioterapeuta $(1,0 \%)$ também compuseram artigos sobre o assunto. Ademais, encontraram-se trabalhos produzidos por acadêmicos do curso de Nutrição $(9,1 \%)$ e autores em que os dados não estavam disponíveis no artigo e não foi encontrado no Currículo Lattes, correspondem a 6,1\% dos resultados.

A respeito da titulação acadêmica dos profissionais, foi observado que 56 autores se encontram com PósGraduação strictu sensu, destes, $30 \mathrm{em}$ grau de Doutor/Doutorado em andamento e 26 em grau de Mestre/Mestrado em andamento. Com relação ao lato sensu, categorizou-se em Especialização, Especialização em andamento e Residência totalizou 23 autores, por ultimo, nota-se a participação de alunos de graduação no desenvolvimento de artigos totalizando 9 autores.

De acordo com Rodrigues GGF, et al. (2017), doutores e mestres são os maiores responsáveis pela autoria dos estudos, ressaltando a atração aos títulos profissionais em virtude destas ganharem valor quando se trata de competência técnica. $O$ autor também afirma que as pós-graduações funcionam em forma de etapas evoluindo de especialidade, mestrado e doutorado e, para tal, parte à busca de novos conhecimentos, resultando em profissionais mais capacitados, consequentemente 0 aumento de publicações. A Tabela 1 figura os dados da formação profissional e titulação acadêmica dos autores.

Tabela 1 - Formação profissional e título acadêmico do primeiro autor dos artigos publicados no período de 2008 a 2018

\begin{tabular}{|c|c|c|c|c|c|}
\hline \multirow[b]{2}{*}{$\begin{array}{l}\text { FORMAÇÃO } \\
\text { PROFISSIONAL }\end{array}$} & \multirow[b]{2}{*}{ n } & \multirow[b]{2}{*}{ Graduação } & \multirow{2}{*}{$\begin{array}{c}\text { Latu Sensu } \\
\text { Especialização/ } \\
\text { Espec. em } \\
\text { andamento/ } \\
\text { Residência }\end{array}$} & \multicolumn{2}{|c|}{ Strictu Sensu } \\
\hline & & & & $\begin{array}{l}\text { Mestrado/ } \\
\text { Mestrado em } \\
\text { andamento }\end{array}$ & $\begin{array}{c}\text { Doutorado/ } \\
\text { Doutorado } \\
\text { em } \\
\text { andamento }\end{array}$ \\
\hline Nutricionista & 78 & 5 & 21 & 25 & 27 \\
\hline Enfermeiro & 3 & - & - & - & 3 \\
\hline Médico & 2 & - & 2 & & - \\
\hline Fisioterapeuta & 1 & - & - & 1 & - \\
\hline $\begin{array}{l}\text { Outros (alunos de } \\
\text { graduação) }\end{array}$ & 9 & - & - & - & - \\
\hline $\begin{array}{l}\text { Dados não encontrado sobre } \\
\text { o autor }\end{array}$ & 6 & - & - & - & - \\
\hline TOTAL & 99 & 5 & 23 & 26 & 30 \\
\hline
\end{tabular}

Fonte: Suzuki QG, et al., 2019. 
Quanto à participação de universitários no campo das investigações científicas, isso se deve ao fato de que em Instituições de Ensino Superior (IES) as práticas educacionais são fundamentadas sobre a tríade ensino, pesquisa e extensão, isto impulsiona os acadêmicos a desenvolverem esse modelo de trabalho conforme critérios de agências de avaliação, o que representa a construção profissional do indivíduo no campo de pesquisa (VIEIRA S, et al., 2018). Cabe esclarecer que, os "Dados sobre o autor não encontrado" representa informações da formação e titulação não disponíveis no artigo publicado, Currículo Lattes não encontrado na busca na Plataforma Lattes ou dado não atualizado em currículo.

$\mathrm{Na}$ área de atuação profissional dos autores, observou-se que $41,1 \%$ representaram professores que desenvolveram artigos sobre o tema durante esses 10 anos, entretanto houve a representatividade de profissionais assistencialistas $(27,8 \%)$ contribuindo e ampliando a gama de produções científicas relacionadas à nutrição para o tratamento do câncer. Conjuntamente, autores que compuseram parte dos artigos encontrados foram profissionais técnicos administrativos (2,2\%), e categoria "outros" $(7,8 \%)$ que corresponde a estudantes de mestrado, servidores públicos atuantes na direção da alimentação escolar, fiscal de vigilância sanitária, profissionais autônomos, diretor de instituição e gestor de pós-graduação. A quantia remanescente resultou em atuação não disponível (21,1\%).

Direcionando apenas aos autores nutricionistas, dentre os 78 autores e subtraindo os que tiveram os dados indisponíveis perfaz o número de 66 autores com dados encontrados por completo. Relacionados à atuação e título acadêmico observou-se que $50,0 \%$, destes são docentes distribuídos em professores com especialização $(6,1 \%)$, professores mestres ou com mestrado em andamento $(21,2 \%)$, professores doutores ou com doutorado em andamento $(22,7 \%)$, e profissionais assistencialistas $(36,4 \%)$, tais, com lato sensu $(18,2 \%)$, mestrado $(9,1 \%)$ e doutorado $(9,1 \%)$. Os demais profissionais nutricionistas $(13,6 \%)$ atuantes em outras áreas também apresentaram titulação de especialista, mestre e doutor, a maioria vinculados em instituições de pesquisa.

Entre as regiões que mais se destacaram na produção de artigos sobre nutrição e tratamento do câncer, a região Sudeste sobressaiu com o maior percentual $(47,5 \%)$ diante das demais regiões, em seguida a região Sul $(27,3 \%)$, Nordeste $(15,2 \%)$, Centro-Oeste $(8,1 \%)$ e Norte $(2,0 \%)$, respectivamente. Entre os estados, o que mais se destacou com números de artigos publicados foi o estado de São Paulo $(24,2 \%)$ localizado na região Sudeste, seguido do Rio Grande do Sul $(20,2 \%)$ como o estado mais representativo da região Sul, Pernambuco $(7,1 \%)$ na região Nordeste, Distrito Federal $(7,1 \%)$ no Centro-Oeste e o Pará $(2,0 \%)$ excepcionalmente à Região Norte.

Nota-se que há uma centralização e a superioridade de artigos científicos publicados representados pela região Sudeste, o oposto se observa para a região Norte do Brasil no qual apresentaram um número raso. Conforme o Relatório Clarivate Analytics sobre as pesquisas científicas realizadas em todo o território brasileiro entre os anos de 2011 a 2016 - empresa americana pioneira no mundo da indexação e análise de citações - a atividade de pesquisa no Brasil é mais desenvolvida em alguns estados e, em especial nos estados da região Sudeste. Dados deste relatório demonstraram que mais de $40 \%$ dos trabalhos brasileiros têm um autor afiliado a uma instituição em São Paulo.

Hipoteticamente, o desenvolvimento científico no Sudeste pode estar agregada à ampla variabilidade e concentração de Instituições de Ensino Superior (IES), programas de pós-graduação e programas apoiados por fundações estaduais de ciência e tecnologia (C\&T), implementados pelas agências incentivadoras locais e federais, como o Conselho Nacional de Desenvolvimento Científico (CNPq), a Coordenação de Aperfeiçoamento de Pessoal de Nível Superior (CAPES) e a Financiadora de Estudos e Projetos (Finep) (SOARES PB, et al., 2016). A Tabela 2, mostra a quantidade de artigos por IES e respectiva região. 
Tabela 2 - Número de artigos por Instituições no período de 2008 a 2018.

\begin{tabular}{|c|c|c|}
\hline Região & INSTITUIÇÃO & $\mathbf{n}$ \\
\hline \multirow{18}{*}{ SUDESTE (47,5\%) } & Centro Universitário UMA & 1 \\
\hline & Faculdades Unidas do Norte de Minas (FUNORTE) & 1 \\
\hline & Pontifica Universidade Católica (PUC) - Campinas & 2 \\
\hline & Universidade de São Paulo (FMUSP) - Ribeirão Preto & 2 \\
\hline & Universidade de São Paulo (USP) & 3 \\
\hline & Universidade do Estado do Rio de Janeiro (UERJ) & 1 \\
\hline & Universidade Estadual de Campinas (UNICAMP) & 1 \\
\hline & Universidade Estadual Paulista (UNESP) & 2 \\
\hline & Universidade Federal de Juiz de Fora (UFJF) & 1 \\
\hline & Universidade Federal de Minas Gerais (UFMG) & 2 \\
\hline & Universidade Federal de São Carlos (UFSCar) & 1 \\
\hline & Universidade Federal de São Paulo (UNIFESP) & 8 \\
\hline & Universidade Federal de Uberlândia (UFU) & 2 \\
\hline & Universidade Federal de Viçosa (UFV) & 1 \\
\hline & Universidade Federal do Estado do Rio de Janeiro (UNIRIO) & 1 \\
\hline & Universidade Federal do Rio de Janeiro (UFRJ) & 1 \\
\hline & Universidade Presidente Antônio Carlos (Unipac) & 1 \\
\hline & Universidade José do Rosário Vellano (Unifenas) & 1 \\
\hline \multirow[t]{9}{*}{ SUL $(27,3 \%)$} & Faculdade da Serra Gaúcha (FSG) & 1 \\
\hline & Universidade Católica de Pelotas (UCPel) & 3 \\
\hline & Universidade de Caxias do Sul (UCS) & 4 \\
\hline & Universidade do Oeste de Santa Catarina (Unoesc) & 1 \\
\hline & $\begin{array}{l}\text { Universidade Federal de Ciências da Saúde de Porto Alegre } \\
\text { (UFCSPA) }\end{array}$ & 2 \\
\hline & Universidade Federal de Pelotas (UFPEL) & 5 \\
\hline & Universidade Federal de Santa Catarina (UFSC) & 2 \\
\hline & Universidade Federal do Paraná (UFPR) & 1 \\
\hline & Universidade Federal do Rio Grande do Sul (UFRGS) & 3 \\
\hline \multirow[t]{6}{*}{ NORDESTE (15,2\%) } & Universidade de Fortaleza (UNIFOR) & 2 \\
\hline & Universidade de Pernambuco (UPE) & 1 \\
\hline & Universidade Estadual do Ceará (UECE) & 3 \\
\hline & Universidade Federal da Bahia (CAT/UFBA) & 1 \\
\hline & Universidade Federal de Pernambuco (UFPE) & 3 \\
\hline & Universidade Federal do Maranhão (UFMA) & 1 \\
\hline \multirow{3}{*}{$\begin{array}{l}\text { CENTRO-OESTE } \\
(\mathbf{8 , 1 \% )}\end{array}$} & Pontifica Universidade Católica (PUC) - Goiás & 1 \\
\hline & Universidade de Brasília - (UnB) & 2 \\
\hline & Universidade Paulista de Brasília (UNIP) - DF Pontifica & 3 \\
\hline \multirow[t]{2}{*}{ NORTE (2,0\%) } & Universidade Federal do Pará (UFPA) & 2 \\
\hline & OUTROS (Hospitais, Clínicas e Institutos) & 26 \\
\hline TOTAL & & 99 \\
\hline
\end{tabular}

Fonte: Suzuki QG, et al., 2019. 
O número de Instituições representadas neste trabalho totalizou 50, constituindo 35 Universidades e 15 hospitais e clínicas vinculadas ou não ao Ensino e Pesquisa. Destas, a IES que mais desenvolveu trabalhos científicos sobre o tema foi a Universidade Federal de São Paulo (UNIFESP), com 8 artigos publicados. Observou- se que os artigos sucedem de autores concentrados nas regiões Sudeste e Sul do Brasil, fato este que pode corroborar a presunção de Soares PB, et al. (2016) da produtividade intelectual da região estar relacionada à variabilidade e concentração de IES, pós-graduação e programas de C\&T.

O relatório da Clarivate Analytics ainda identificou as 20 instituições mais prolíficas. Destas, 15 são universidades federais e 5 estaduais. Em 2011, a Fundação de Amparo as Pesquisas de São Paulo (FAPESP) apresentou dados da produção nacional de 2003 - 2012 de contribuições das Universidades brasileiras que das 20 universidades, 14 eram federais e 6 estaduais (SANTOS SM, 2015). Isto atesta que universidades públicas produzem maciçamente e alcançam bons números entre os artigos mais citados do mundo. Conforme Chiarini T e Vieira KP (2012), as instituições de ensino superior são o maior suporte do sistema de pesquisa do país, principalmente aos programas de pós-graduação e o principal locus de produção de conhecimento vem de IES federais.

O sistema universitário do Brasil de IES privado é dedicado ao ensino e com atividade de pesquisa para o que remanesce, ou seja, preocupa-se quase que tão-somente com o ensino e cumpre as demais atividades acadêmicas na medida de sua obrigatoriedade, onde a maioria não dispõe de uma infraestrutura qualificada para atender suas necessidades da pesquisa cientifica (CHIARINI T e VIEIRA KP, 2012; SOCZEK D e ALENCASTRO $M, 2012$ ). A dedicação à pesquisa científica não é frequente, ficando a produção de conhecimento científico a cargo das universidades públicas (CHIARINI T e VIEIRA KP, 2012).

A produção das instituições assistenciais representou 26,3\%, o que inclui institutos especializados, clínicas, hospitais públicos e particulares correspondendo a menor frequência de participações. As publicações de IES, sejam públicas ou privadas, revelaram $(73,7 \%)$ a produção majoritária de artigos científicos sobre a temática muito embora haja a participação das universidades privadas $(27,4 \%)$, estas, representam uma contribuição inferior quanto ao fomento à pesquisa investido pelas instituições públicas aceirando o que diz Soczek D e Alencastro M (2012) sobre a produção acadêmico-científica das IES particulares apresentar uma representatividade baixa.

Neste panorama de produções científicas por IES, Hilu L e Gisi ML (2011) explicam que grande parte das pós-graducação se encontram em Universidades Públicas e poucos são os investimentos nas IES privadas para programas stricto sensu, pois estas dependem de mensalidades e a pesquisa científica exige um alto custo, o que requer grandes investimentos. Além disso, acrescenta a concorência na busca de entidades de fomento entre as IES privadas e públicas que na maioria dos casos as universidades públicas lucram os recursos por terem perfil fundamentado em pesquisas.

Em se tratando de periódicos, 42 periódicos foram verificados e, a Revista Brasileira de Cancerologia obteve ênfase em artigos publicados sobre o assunto contemplando $20,2 \%$ dos trabalhos elaborados, logo a Nutrición Hospitalaria junto com a Revista de Nutrição com 10,1\% das publicações. A Revista Brasileira de Cancerologia é um periódico do Instituto Nacional de Câncer José Alencar Gomes da Silva - INCA, cuja finalidade é divulgar conteúdos acerca do controle e tratamento do câncer, a Nutrición Hospitalaria faz parte de uma Rede de Revistas Científicas da América Latina e Caribe, Espanha e Portugal e a Revista de Nutrição é um periódico especializado na área da Nutrição, todos com acesso aberto. A Tabela 3 apresenta as revistas que publicaram dois ou mais artigos no período de 10 anos. 
Tabela 3 - Número de artigos publicados por periódico acima de dois artigos e número de publicações por ano no período de 2008 a 2018.

\begin{tabular}{|c|c|c|c|c|c|c|c|c|c|c|c|c|}
\hline PERIÓDICOS & 2008 & 2009 & 2010 & 2011 & 2012 & 2013 & 2014 & 2015 & 2016 & 2017 & 2018 & $\mathbf{N}$ \\
\hline $\begin{array}{l}\text { ABCD Arquivos } \\
\text { Brasileiros de } \\
\text { Cirurgia Digestiva }\end{array}$ & - & - & - & - & 1 & - & - & 1 & 1 & - & - & 3 \\
\hline $\begin{array}{l}\text { Arquivos de } \\
\text { Gastroenterologia }\end{array}$ & - & - & 1 & 1 & 1 & - & 1 & - & - & 1 & - & 5 \\
\hline BRASPEN JOURNAL & - & - & - & - & - & - & - & - & - & 2 & 1 & 3 \\
\hline $\begin{array}{l}\text { Ciência \& Saúde } \\
\text { Coletiva }\end{array}$ & - & - & - & - & - & - & 1 & 1 & 1 & - & - & 3 \\
\hline $\begin{array}{l}\text { Comunicação em } \\
\text { ciências da saúde }\end{array}$ & 1 & - & - & - & - & 2 & - & - & - & - & - & 3 \\
\hline $\begin{array}{l}\text { Journal of the Health } \\
\text { Sciences Institute }\end{array}$ & - & - & - & - & 1 & - & - & - & - & 1 & - & 2 \\
\hline Nutrición Hospitalaria & 1 & - & - & - & 5 & 3 & - & - & - & 1 & - & 10 \\
\hline $\begin{array}{l}\text { Nutrire: Revista } \\
\text { Sociedade Brasileira } \\
\text { de Alimentação e } \\
\text { Nutrição }\end{array}$ & - & - & 1 & - & - & 1 & - & - & - & - & - & 2 \\
\hline $\begin{array}{l}\text { Nutrition in Clinical } \\
\text { Practice }\end{array}$ & - & - & - & - & - & - & - & - & - & 2 & - & 2 \\
\hline Nutrition Journal & - & - & - & - & - & - & - & 1 & - & 1 & - & 2 \\
\hline $\begin{array}{l}\text { Revista Brasileira de } \\
\text { Nutrição Clínica }\end{array}$ & 2 & - & - & - & - & - & - & - & - & - & - & 2 \\
\hline $\begin{array}{l}\text { Revista Brasileira de } \\
\text { Cancerologia }\end{array}$ & - & - & - & - & 5 & 2 & 1 & 5 & 3 & - & 4 & 20 \\
\hline $\begin{array}{l}\text { Revista Brasileira em } \\
\text { Promoção da Saúde }\end{array}$ & - & - & - & - & - & 1 & - & - & - & 1 & - & 2 \\
\hline Revista de Nutrição & - & 2 & 4 & - & 1 & - & - & - & 1 & 2 & - & 10 \\
\hline Revista HCPA & - & - & - & 2 & 1 & - & - & - & - & - & - & 3 \\
\hline TOTAL & 4 & 2 & 6 & 3 & 15 & 9 & 3 & 8 & 6 & 11 & 5 & 72 \\
\hline
\end{tabular}

Fonte: Suzuki QG, et al., 2019. 
Guedes VLS (2012) e Júnior CM, et al. (2016), em seus trabalhos sobre bibliometria dizem que na Lei de Bradford, onde é possível avaliar os níveis de interesse das revistas em um campo da ciência, os periódicos com grandes quantidades de artigos sobre um tema compõem o núcleo e, possivelmente de alta qualidade e relevância para a área pesquisada. Neste seguimento, Júnior CM, et al. (2016) também explica que as primeiras produções sobre determinado assunto estão contidas em um número limitado de periódicos, isso faz com que a aceitação e publicação destes artigos impulsionem outros autores deste mesmo tema a encaminhar suas produções cientificas para os periódicos que estão presentes no núcleo.

Conforme a formulação da Lei de Bradford pode ser formada um número variável de zonas de produtividade que são compostas basicamente de três: alta frequência ou núcleo, média e de baixa frequência ou de dispersão. Logo depois foi levantada por outros autores, como Vickery, em 1948 e Goffman e Warren no ano de 1969, a possibilidade da ampliação do número de zonas, quando adotaram a divisão no número máximo de zonas (RIBEIRO LVP, et al., 2013; PINHEIRO LVR, 1983).

$\mathrm{Na}$ análise dos periódicos sob óptica da Lei de Bradford, os 42 periódicos que somam os 99 artigos do trabalho, observa-se que o núcleo está representado apenas por um periódico com 20 publicações e de conteúdo sobre a temática em estudo, concomitante ao que propõe a Lei de que os periódicos que integram o núcleo serem os mais prolíficos. A zona 1 apresenta os 3 periódicos que somaram os 25 artigos encontrados, entre eles dois se caracterizam como periódico da área da nutrição. Em seguida, a zona 2 e 3 , mais dispersa, é visto em cada uma, o aumento na quantidade de periódicos e a redução do número de artigos, conforme Figura 1.

Figura 1 - Apresenta as zonas com a quantidade de periódicos e seus respectivos números de artigos.

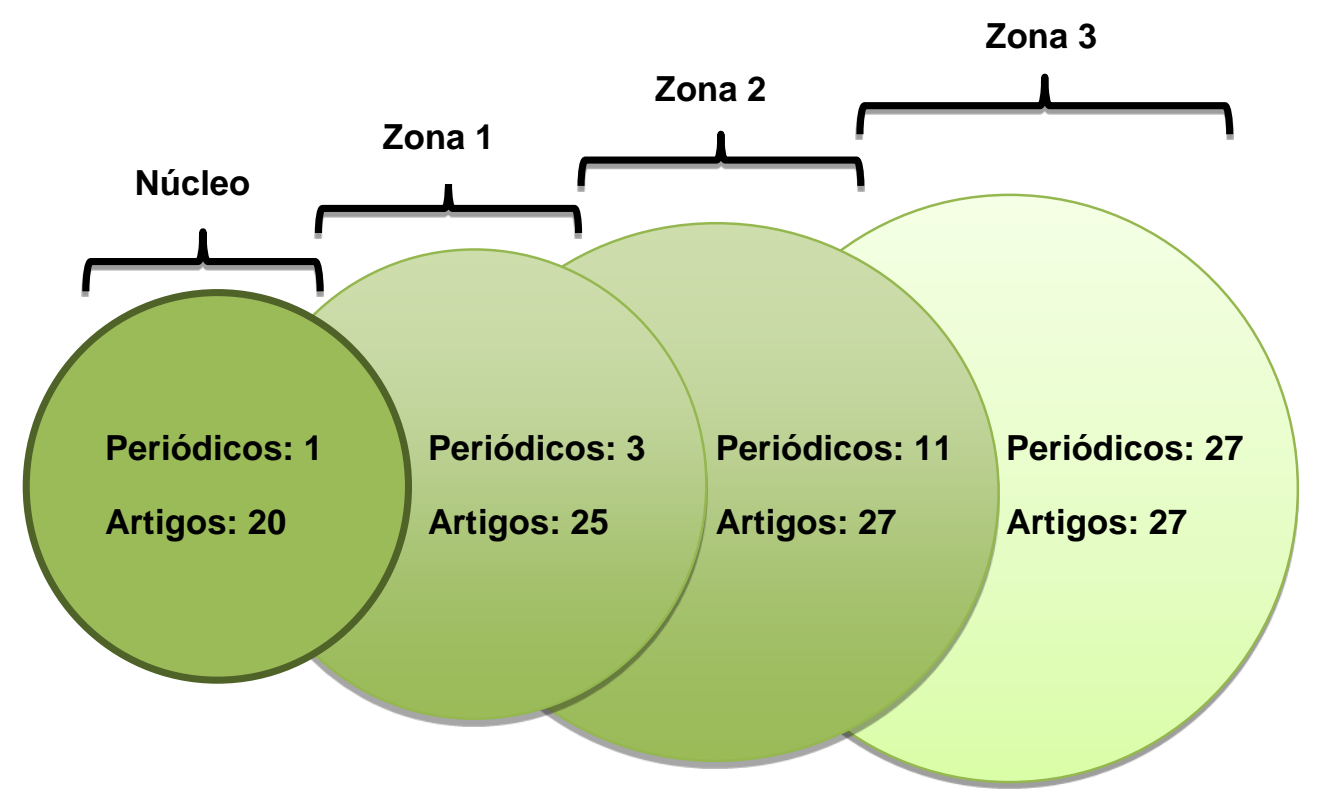

Fonte: Suzuki QG, et al., 2019.

Os assuntos que mais se destacaram nos artigos foram referentes ao estado nutricional ou risco nutricional de pacientes oncológicos $(44,4 \%)$, os impactos de terapias nutricionais a partir da imunonutrição ou de pacientes sob uso de dietas enterais $(16,2 \%)$, métodos de triagem nutricional $(13,1 \%)$ e consumo alimentar $(9,1 \%)$. Logo, nota-se que a preocupação com a condição física e nutricional, aparenta ser as principais linhas de pesquisa da nutrição sobre essa patologia, devido a deficiência no estado nutricional comprometer a recuperação dos pacientes oncológicos em procedimentos cirúrgicos ou em tratamento antineoplásico (OLIVEIRA AL e AARESTRUP FM, 2012; PINTO AS, et al., 2015). 
Relacionado ao processo patológico neoplásico, artigos voltados para pacientes com tumor em específico, o câncer do trato gastrointestinal $(19,2 \%)$ foi o mais discorrido entre as publicações, em seguida, câncer infanto-juvenil $(17,2 \%)$, câncer de mama $(15,2 \%)$ e cabeça e pescoço $(9,1 \%)$. Outros escreveram sobre as neoplasias de uma maneira geral $(35,4 \%)$, três ou mais tipos de câncer encontrado em um único trabalho e os tipos de neoplasias tratados nesses artigos foram os que se desenvolvem no trato gastrointestinal, mama, cabeça e pescoço, pulmão, tórax, próstata, metastático, sarcomas, hematológicos, adenocarcinoma, linfoma e leucemia, geniturinário, ósseo, pele, mieloma, coluna e linfático.

Referente a Classificação Qualis, foram identificados os estratos Qualis dos 42 periódicos encontrados. A maioria parte de trabalhos publicados em periódicos de estrato Qualis B1 a B2 (33,3\%), em seguida, Qualis B3 a B5 (23,8\%), Qualis A1 a A2 (7,1\%) e Qualis C (7,1\%). Houve revistas que apresentaram mais de um Qualis em diversas áreas de avaliação $(19,0 \%)$ e Qualis não encontrado $(9,5 \%)$, no qual não foi possível verificar o estrato de classificação na Plataforma Sucupira que disponibiliza o nível de evidência de periódicos.

A Tabela 4 apresenta a classificação Qualis dos periódicos.

Tabela 4 - Periódicos segundo estrado de classificação QUALIS, no período de 2008 a 2018.

\begin{tabular}{lcc}
\hline Qualis & N & $\%$ \\
\hline Qualis A1 e A2 & 3 & 7,1 \\
Qualis B1 a B2 & 14 & 33,3 \\
Qualis B3 a B5 & 10 & 23,8 \\
Qualis C & 3 & 7,1 \\
Revistas com mais de um Qualis & 8 & 19,0 \\
Qualis não encontrado & 4 & 9,5 \\
\hline Total & $\mathbf{4 2}$ & $\mathbf{1 0 0}$ \\
\hline
\end{tabular}

Fonte: Suzuki QG, et al., 2019.

No que se refere aos descritores e palavras-chave foram identificadas 441 palavras conectadas aos resumos analisados, destes, 216 estavam associados à nutrição e ao câncer. Os mais citados foram: estado nutricional (11,3\%), avaliação nutricional $(5,4 \%)$, desnutrição $(4,3 \%)$ e terapia enteral $(2,0 \%)$ para a nutrição e neoplasia $(12,5 \%)$, câncer $(6,8 \%)$ e oncologia $(1,4 \%)$ para a patologia.

Desenvolver pesquisa científica na área da Nutrição possibilita atingir conhecimentos não apenas ao que parte do próprio campo, mas permite abranger outras diferentes áreas. Com investimentos na ciência, a qualidade e a expectativa de vida se elevaram e isso conduziu produções intelectuais que pudessem explicar as condições nutricionais encontradas em portadores de doenças crônicas e degenerativas que por vezes estão associadas às mudanças no consumo dos novos alimentos.

\section{CONSIDERAÇÕES FINAIS}

A nutrição garante suporte essencial durante o tratamento do câncer, após o diagnóstico a intervenção alimentar individualizada proporciona a recuperação e manutenção do estado nutricional. Esse conhecimento é embasado através de produções científicas disponíveis que comprovam o argumento, quanto mais se produz conhecimento escrito mais o conhecimento é disseminado à sociedade. Desta maneira, pesquisas sobre a nutrição no tratamento do câncer expõe o quanto o estudo é importante para descoberta de novos meios de cuidados a fim de melhorar a qualidade de vida. 


\section{REFERÊNCIAS}

1. ABRAHÃO SAB, MACHADO EC. Suplementação de glutamina no tratamento de pacientes com câncer: uma revisão bibliográfica, Goiânia. Estudos, 2014; 41(2): 215-222.

2. ARAÚJO CA. Bibliometria: evolução histórica e questões atuais. Em Questão, 2006; 12(1): 11-32.

3. AZEVEDO CD, DAL BOSCO SM. Perfil nutricional, dietético e qualidade de vida de pacientes em tratamento quimioterápico. ConScientiae Saúde, 2011; 10(1): 23-30.

4. BARRETO ML. O desafio de avaliar o impacto das ciências para além da bibliometria. Rev. Saúde Pública, 2013; 47(4): 834-837.

5. BRASIL. Ministério da Saúde. Instituto Nacional de Câncer José Alencar Gomes da Silva. Estimativa 2018: incidência de câncer no Brasil. Rio de Janeiro. INCA, 2017.

6. BUONO HCD, et al. A importância do nutricionista no tratamento de pacientes oncológicos. Revista Saúde em Foco, 2017 - Edição no 9.

7. CARVALHO G, et al. Qual a relevância da nutrição em oncologia? Acta Med Port, 2011; 24(S4): 1041-1050.

8. CHIARINI T, VIEIRA KP. Universidades como Produtoras de Conhecimento para o Desenvolvimento Econômico: Sistema Superior de Ensino e as Políticas de CT\&I. RBE, 2012; 66(1): 117-132.

9. Clarivate Analytics CROSS D, et al. Research in Brazil: A report for CAPES by Clarivate Analytics. Clarivate Analytics, 2018.

10. DEMO G, et al. Resiliência no trabalho: Revisão bibliométrica sistemática no contexto brasileiro e itinerários da produção nacional. Revista Psicologia: Organizações e Trabalho, 2017; 17(3): 180-189.

11. DUTRA IKA, SAGRILLO MR. Terapia nutricional para pacientes oncológicos com caquexia. Disciplinarum Scientia. Série: Ciências da Saúde, 2014; 15(1): 155-169.

12. FUNDAÇÃO DE AMPARO À PESQUISA DO ESTADO DE SÃO PAULO. Indicadores de Ciência, Tecnologia e Inovação em São Paulo 2010. São Paulo: Fapesp, 2010.

13. GARÓFOLO A, et al. Dieta e câncer: um enfoque epidemiológico. Rev. Nutr., 2004; 17(4): 491-505.

14. GUEDES VLS. A bibliometria e a gestão da informação e do conhecimento científico e tecnológico: uma revisão da literature. PontodeAcesso, Salvador, V.6, n.2 ,p. 74-109 ago 2012.

15. HILU L, GISI ML. Produção científica no Brasil: um comparativo entre universidades públicas e privadas. In: Anais do 10 Congresso Nacional de Educação. 1 Seminário Internacional de Representações Sociais, Subjetividade e Educação. Curitiba: Champagnat; 2011. p. 5665-72.

16. JúNIOR CM, et al. As leis da bibliometria em diferentes bases de dados científicos. Revista de Ciências da Administração, 2016; 18(44): 111-123.

17. OLIVEIRA AL, AARESTRUP FM. Avaliação nutricional e atividade inflamatória sistêmica de pacientes com câncer colorretal submetidos à suplementação com simbiótico. ABCD Arq Bras Cir Dig., 2012; 25(3): 147-153.

18. PINHEIRO LVR. Lei de bradford: uma reformulação conceitual. Ci. Inf., 1983; 12(2): 59-80.

19. PINTO AS, et al. Abreviação do jejum entre pacientes submetidos à cirurgia oncológica: revisão sistemática. $A B C D$ Arq Bras Cir Dig, 2015; 28(1): 70-73.

20. RIBEIRO LVP, et al. Desbravando caminhos de navegantes do portal Canal Ciência via metrias de informação. Liinc em Revista, 2013; 9(1): 237-254.

21. RODRIGUES GGF, et al. Cuidados paliativos direcionados ao cliente oncológico: estudo bibliométrico. Rev enferm UFPE online, 2017; 11(Supl. 3):1349-56.

22. SANTOS GC. Análise Bibliométrica dos Artigos Publicados como Estudos Bibliométricos na História do Congresso Brasileiro de Custos. Pensar Contábil, 2015; XVII (62): 4-13.

23. SANTOS SM. Desempenho das universidades brasileiras nos rankings internacionais: áreas de destaque da produção científica brasileira. Tese (Doutorado em Ciência da Informação) - Escola de Comunicações e Artes. Universidade de São Paulo, São Paulo, 2015; 344 p.

24. SILVA FQ, et al. Estudo bibliométrico: orientações sobre sua aplicação. Revista Brasileira de Marketing - ReMark, 2016; 15(2): 246-262.

25. SOARES PB, et al. Análise bibliométrica da produção científica brasileira sobre Tecnologia de Construção e Edificações na base de dados Web of Science. Ambiente Construído, 2016; 16(1): 175-185.

26. SOCZEK D, ALENCASTRO M. Pesquisa acadêmica em instituições de ensino superior particulares: desafios e perspectivas. Revista Intersaberes, 2012; 7(13): 46-66.

27. VIEIRA S, et al. O que podemos aprender com os Trabalhos de Conclusão de Curso de graduação em Odontologia? Um estudo bibliométrico. Revista da ABENO, 2018; 18(3): 2-11. 